$2 \mathrm{I}-\mathrm{cm}$ observations of other galaxies far from the central regions refer to matter in the haloes. It may well be disk matter. From the evidence of the neutral hydrogen in our Galaxy at high galactic latitude one would expect a large velocity dispersion in the haloes of other galaxies as well. This is not shown by the present observations.

\title{
References
}

[1] Symposium No. I of the I.A.U. Co-ordination of Galactic Research, ed. A. Blaauw (Cambridge University Press, 1955), p. 4.

[2] Iriarte, B. and Chavira, E. Bol. Obs. Tonantzintla y Tacubaya, no. I6, 3, 1957.

[3] Baldwin, J. E. Symposium No. 4 of the I.A.U. Radio Astronomy, ed. H. C. van de Hulst (Cambridge University Press, 1957), p. 233.

[4] Bolton, J. E. and Westfold, K. C. Austr. J. Sci. Res. A 3, 19, 1950.

[5] Dröge, F. and Priester, W. Z. f. Astroph. 40, 236, 1956.

[6] Mills, B. Y. Austr. J. Phys. 8, 368, 1955.

[7] Westerhout, G. Symposium No. 4 of the I.A.U. Radio Astronomy, ed. H. C. van de Hulst (Cambridge University Press, 1957), p. 26.

[8] Shain, C. A. Mt Stromlo Pre-conference on Co-ordination of Galactic Research, p. 53, Canberra, 1957.

[9] Mills, B. Y. Radio Frequency Radiation from External Galaxies, Handbuch der Physik, in press.

[10] Sklovsky, I. S. Dok. Akad. Nauk S.S.S.R. 90, 983, 1953.

[11] Baldwin, J. E. Nature, 174, 320, 1954.

[12] Seeger, Ch. L., Westerhout, G. and Conway, R. G. Astroph. J. 126, 585, 1957.

[13] Hulst, H. C. van de, Raimond, E. and Woerden, H. van. Bull. Astr. Inst. Netherl. 14, $1,1957$.

[14] Kerr, F. J., Hindmann, J. V. and Robinson, B. J. Austr. J. Phys. 7, 297, 1954.

\section{(D) THE NUGLEAR REGION}

The nuclear region may be explored by means of both radio and optical data. Optical methods include surveys of variable stars and various other objects, but of special interest are measurements in the far infra-red, which may provide a connexion between optical and radio observations.

\section{Variable stars}

\section{(I) Optical investigations}

Surveys of variable stars in three selected fields in the nuclear region were reported by Oosterhoff. The centres of the fields are

$\begin{array}{lll} & l & b \\ \text { Sgr I } & 329^{\circ} \cdot 1 & -4 \cdot 0 \\ \text { Sgr II } & 33^{1 \cdot 7} & -6 \cdot 6 \\ \text { CPD }-31^{\circ} 5547 & 329 & -10\end{array}$


The first two fields were selected by Baade to supplement the investigation of a previously selected field in the central region, the results of which have recently been published by Gaposchkin [1]. Most of the plates of these two fields, which cover areas of $0.60 \times 0^{\circ} 45$ with limiting magnitude 19 , were taken with the Radcliffe reflector. Some plates of Sgr II were taken by Baade with the roo-inch reflector at Mount Wilson. The third field, investigated by means of the Franklin-Adams camera, covers $10^{\circ} \times 10^{\circ}$ and has limiting magnitude about $16 \cdot 0$. The total number of plates of the three fields are about 90,110 , and 300 , respectively. The investigation of the plates was started by the late Mr Schuurman and has been continued by Mr Ponsen.

Although only part of the plates have been blinked as yet I 55 variables have been found in the field Sgr I. This number is probably approximately complete, and for the eighty-one variables which have been estimated the distribution over different types is as follows:

$\begin{array}{lr}\text { RR Lyrae type } & 30 \\ \text { Long-period } & 22 \\ \text { Semi-regular } & 17 \\ \text { Eclipsing binaries } & 4 \\ \text { Uncertain } & 8\end{array}$

The last group, which will be further investigated, probably contains a few Cepheids. During the further investigation the relative number of $R R$ Lyrae stars and semi-regular variables may well increase considerably, as many of them have small amplitudes. Until now only few periods have been derived; they confirm the well known tendency of the long-period variables in the nuclear region to show periods below 250 days, the majority may perhaps even have periods below 150 days. As for the RR Lyrae variables, both periods of 0.3 day and of 0.5 day and longer are represented. No light-curves are available as yet. From a first inspection of the plates, the interstellar absorption in Sgr I seems far from homogeneous.

In the field Sgr II, situated somewhat further from the galactic centre, about fifty variables have been found, but there are probably some more to be discovered. Forty-eight variables have been classified; they are distributed as follows:

$\begin{array}{lr}\text { RR Lyrae type } & 23 \\ \text { Long-period and semi-regular } & 20 \\ \text { Eclipsing binaries } & 1 \\ \text { Uncertain } & 4\end{array}$

For five long-period and semi-regular variables and for twenty-one $\mathbf{R} \mathbf{R}$ Lyrae variables periods could be derived. Only one star in the long-period group has a period longer than 250 days; in the RR Lyrae group the 
majority has periods about 0.5 day or longer, but there are several with periods around 0.3 day.

Both in Sgr I and II the frequency maximum of the RR Lyrae stars is found at about magnitude 16 or 17 . The same thing was very pronounced in Baade's field close to the centre [1]. In all three cases it is therefore to be assumed that the variables really belong to the nuclear region and that the investigations have penetrated the central bulge. The nature of the variables seems to change between the three fields. In Baade's field a large number of very short-period RR Lyrae variables were found (periods below 0.4 day). Even if some of the periods are spurious this fact seems to be established. In the field Sgr II the periods of the RR Lyrae variables are longer, and probably Sgr I will prove an intermediate case in this respect.

In the third field mentioned above, CPD $-31^{\circ} 9547$, which has much larger field, a systematic survey for long-period variables is in progress. The first plate pair yielded 146 variables of this type, the majority of them being new objects. $90 \%$ of these stars has maximum brightness between 13 and I 4.5 photographic magnitude. The number of RR Lyrae variables is only nine, owing to their lower absolute magnitude. There is little doubt that the numerous long-period and semi-regular stars belong to the outskirts of the central galactic bulge. Although it is difficult to give an accurate estimate, the total number or these stars in the area is considered by Ponsen to be about 1000, thus forming valuable material for a statistical investigation.

In this connexion it is of interest that Oosterhoff [2] has recently investigated the population characteristics of the Cepheids in a region $70^{\circ} \times 70^{\circ}$ around the nucleus. Most of the Cepheids in this area are situated south of the galactic equator, a distribution which is quite different from that of other stars. In order to make a statistical separation of Cepheids of population I and population II the frequency distribution of the periods was studied, and from comparisons with statistics for pure samples of the two populations it was found that the Cepheids brighter than magnitude Io or I 2 within the belt $\pm 4^{\circ}$ latitude give a frequency distribution quite similar to that of the normal population I Cepheids. For all the others the frequency distribution is nearly the same as that of the Cepheids in the globular clusters. The number of Cepheids studied was about 120 and it thus seems that about forty of the brighter objects are population I Cepheids probably associated with a spiral arm in the direction of the centre, while the others are typical population II objects scattered around the galactic nucleus.

The infra-red spectral surveys reported by Nassau have disclosed a large number of red variables in the nuclear region. The area around the 
globular cluster NGC 6522 has been especially studied, partly by means of photometric material provided by Baade and by Haro. The frequency maximum of the variables is found at about 12th magnitude infra-red. Most of the stars appear to be of rather late spectral class and the majority probably belong to the semi-regular or irregular type of variables.

If there is a clustering of variable stars of various types in the galactic nucleus, as seems probable, it is of greatest importance to investigate the magnitude differences, thus the differences in absolute magnitudes, between the irregular and semi-regular variables on the one hand and the long-period variables with relatively short periods and the RR Lyrae stars on the other.

\section{Survey of $M$ giants}

The Cleveland infra-red spectral surveys show that the non-variable $M$ stars are distributed in about the same way as the red variables. In the nuclear region there appear to be relatively more stars of late spectral classes than in other regions investigated.

\section{Planetary nebulae and novae}

As is well known the planetary nebulae and novae show a concentration towards the galactic nucleus. However, according to Baade the concentration towards the disk of the Galaxy is more significant and the distribution of these objects is therefore discussed in the section dealing with the disk of the Galaxy.

\section{Infra-red continuum surveys}

Surveys of the infra-red radiation at a wave-length of about $\mathrm{I} \mu$ from the nuclear region have been made by Stebbins and Whitford [3], by Kaliniak, Krassovsky, and Nikonov [4] and by Dufay and his collaborators [5]. The results seem, however, still to be mixed up with absorption effects to an extent that it is difficult to distinguish between absorption and real structure. In order to obtain more definitive results it is necessary to go further into the infra-red than has been done so far. It was reported at the conference that experiments at about $15 \mu$ are in progress at the Naval Research Laboratory in Washington. Whitford was also reported to have started work at about ro $\mu$. There is an entirely clear window in the atmosphere in this region, but it is very difficult to construct receivers which are sensitive enough. These infra-red surveys are important also because they provide a bridge between the optical and radio investigations. 


\section{(2) Radio Observations}

Until recently most of the continuum surveys of the nuclear region have been made with aerials having beam-widths larger than $8^{\circ}$. Westerhout reported that only six surveys have been published which give enough detail to distinguish discrete sources in this region. In addition, two single observations have been made at very short wave-lengths. At these short wavelengths, the main feature in the direction of the galactic centre is a discrete source with a diameter of 0.25 to $0^{\circ} \cdot 50$, designated as source no. 5 by Haddock, Mayer and Sloanaker [6], which is superposed on a broader source with a diameter of about $2^{\circ}$, designated no. 9 . The combination of these two sources is clearly different from the underlying galactic ridge, which has roughly the same width and intensity over a region covering a longitude interval of $40^{\circ}$ around the centre.

At the longer wave-lengths the situation is more confused, as the galactic ridge begins to bulge out in latitude, and also the intensity of the ridge rises slowly towards the centre. However, from Mill's survey [7] at $3.5 \mathrm{~m}$ it is evident that in the direction of the centre a source having the width of about $3^{\circ}$ is superposed on the ridge.

The available information is summarized in Table 3 . The first column gives the reference number, the second the wave-length and the third the width of the antenna beam between half-power points. In the fourth column the half-width of the central source is given, measured above the background, in two co-ordinates, while aerial temperatures and flux densities are found in the fifth and sixth columns. The values of the flux densities are very uncertain, as it is difficult to decide which part of the central maximum should be considered the central source. A factor of two can easily be allowed for.

It is interesting to compare the $22 \mathrm{~cm}$ and $3.5 \mathrm{~m}$ results. At $3.5 \mathrm{~m}$ Mills [7] observes a minimum at the exact position of the $22 \mathrm{~cm}$ source. This minimum can be readily explained by assuming that the source at $22 \mathrm{~cm}$ consists of ionized hydrogen.

Correcting for beam-width we find a maximum temperature at $22 \mathrm{~cm}$ of roughly $300^{\circ} \mathrm{K}$ corresponding to an optical depth of 0.03 . At $3.5 \mathrm{~m}$ the optical depth then would be about 6 . Even at a distance of $I^{\circ}$ from the centre, where the temperature is about $50^{\circ} \mathrm{K}$, we have an optical depth of 0.005 at $22 \mathrm{~cm}$ and $\mathrm{I}$ at $3.5 \mathrm{~m}$. From this we may conclude that over a range of $2^{\circ}$ in longitude practically all radiation from behind the centre is blotted out. If the high-temperature maximum, observed at longer wavelengths, is due to a central concentration of matter, radiating non- 
Table 3. Radio emission from the galactic centre

\begin{tabular}{|c|c|c|c|c|c|c|}
\hline $\begin{array}{l}\text { Reference } \\
\text { number }\end{array}$ & $\begin{array}{l}\text { Wave- } \\
\text { length } \\
\text { in } \mathrm{cm}\end{array}$ & $\begin{array}{l}\text { Beam- } \\
\text { width }\end{array}$ & $\begin{array}{l}\text { Half-width } \\
\text { of source }\end{array}$ & $\begin{array}{c}T_{b} \\
\left({ }^{\circ} \mathrm{K}\right)\end{array}$ & $\begin{array}{l}\text { Flux } \\
\text { density } \\
\times 10^{24}\end{array}$ & Remarks \\
\hline I & 3 & $0{ }^{1}{ }_{13} \times 00_{15}$ & $0 \div 25$ & ? & $4-5$ & $\begin{array}{l}\text { Small source (no. 5) } \\
\text { only }\end{array}$ \\
\hline 2 & 9.4 & $0.45 \times 0.40$ & Point & I $7\left(=T_{a}\right)$ & $4 \cdot 8$ & $\begin{array}{l}\text { No. } 5 \text { only. Broad } \\
\text { source (no. 9) has } \\
T_{a}=6^{\circ}, T_{b}=10^{\circ} \mathrm{K}\end{array}$ \\
\hline 3 & 21 & 0.92 & ? & $45-65\left(=T_{a}\right)$ & 17 & $\begin{array}{l}\text { No. } 5+\text { no. } 9 \text { (no. } 9 \\
\left.\text { has } T_{b}=6^{\circ}-30^{\circ}\right)\end{array}$ \\
\hline 4 & 22 & 0.57 & $0.83 \times 0.64$ & 166 & 35 & $\begin{array}{l}\text { Integration over nos. } 5 \\
\text { and } 9\end{array}$ \\
\hline 5 & 33 & $3 \times 6$ & Point & 29 & 29 & No. $5+$ no. 9 \\
\hline 6 & 50 & $3 \cdot 3$ & Point & 40 & 29 & No. $5+$ no. 9 \\
\hline $\begin{array}{l}7 \\
8\end{array}$ & $\begin{array}{l}75 \\
75\end{array}$ & $\begin{array}{l}2 \\
2\end{array}$ & $\begin{array}{l}\text { Point } \\
\text { Point }\end{array}$ & $\begin{array}{l}310 \\
180\end{array}$ & $\begin{array}{l}33 \\
16 \cdot 4\end{array}$ & $\begin{array}{l}\text { No. } 5+\text { no. } 9 \\
\text { No. } 5+\text { no. } 9 \text { ? }\end{array}$ \\
\hline 9 & $\begin{array}{r}15 \\
350\end{array}$ & 0.92 & ? & 32,500 & - & $\begin{array}{l}\text { Source seen in ab- } \\
\text { sorption. 'Back- } \\
\text { ground' } 5.5 \times 10^{4} \text { ? }\end{array}$ \\
\hline 10 & 1500 & $1 \cdot 4$ & $2 \cdot 4$ & 200,000 & - & $\begin{array}{l}\text { Source seen in ab- } \\
\text { sorption. 'Back- } \\
\text { ground' } 5 \times 10^{5} \text { ? }\end{array}$ \\
\hline
\end{tabular}

References to the table

1. Haddock, F. T. and McCullough, T. P. Astron. J. 60, 16I, 1955.

2. Haddock, F. T., Mayer, C. H. and Sloanaker, R. M. Nature, 174, 176, 1954.

3. Hagen, J. P., Lilley, A. E. and McClain, E. F. Astroph. J. 122, 361, 1955.

4. Westerhout, G. C.R. 245, 35, 1957.

5. Denisse, J. F., Leroux, E. and Steinberg, J. L. C.R. 240, 278, 1955. See also reference no. 6.

6. Piddington, J. H. and Trent, G. H. Austr. J. Phys. 9, 74, 1956.

7. Westerhout, G. Bull. Astr. Inst. Netherl. 13, 105, 1956.

8. McGee, R. X., Slee, O. B. and Stanley, G. J. Austr. J. Phys. 8, 347, 1955.

9. Mills, B. Y. Observatory, 76, 65, 1956.

10. Shain, C. A. Austr. J. Phys. 10, 195, 1957.

thermally, this might mean that the ionized hydrogen region is situated in the centre of the galactic system. The $32,500^{\circ} \mathrm{K}$ observed at $3.5 \mathrm{~m}$ in this direction is then partly due to foreground radiation $\left(22,500^{\circ} \mathrm{K}\right)$ and partly to black-body radiation from the thermal source $\left(10,000^{\circ} \mathrm{K}\right)$. In that case the source has blotted out $22,500^{\circ} \mathrm{K}$ from behind so that the peak temperature in the absence of the ionized hydrogen should have been $45,000^{\circ} \mathrm{K}$.

Davies and Williams [8] and McClain [9] have observed the central source in absorption at $2 \mathrm{I} \mathrm{cm}$ and conclude from their observations that it must be situated at $3 \mathrm{kpc}$ from the sun. The initial data and the consequent interpretation are, however, very uncertain.

Substantial evidence for the source being the galactic nucleus itself has recently been obtained by van Woerden, Rougoor, and Oort [10]. In the direction of the centre they observed a maximum in $21 \mathrm{~cm}$-line profiles, 
attributable to a small spiral arm, with a radial velocity of $-50 \mathrm{~km} / \mathrm{sec}$. It is seen as an absorption feature at the position of the centre source, indicating that it is lying in front of this source. If the source was situated at a distance of only $3 \mathrm{kpc}$ from the sun, the small spiral arm would be lying in a region where thus far only small deviations from circular velocity have been observed. On the other hand, in the neighbourhood of the galactic centre, $21-\mathrm{cm}$ line measurements show deviations from circular motion as large as $200 \mathrm{~km} / \mathrm{sec}$. They conclude that the source is situated in the central region and is probably to be identified with the nucleus of our Galaxy.

As regards the expansion observed in the nuclear region, Paranago [11] in a study of the K-effect has shown that some sub-systems of the Galaxy may be expanding while others are contracting. An alternative view was put forward by Lindblad. He suggested that perhaps there are some similarities between the galactic system and the barred spirals, where we have a very small nucleus from which threads of dark matter extend and where we very often find a small spiral in the centre of the system.

It is obvious that in the direction of the galactic centre optical measurements, particularly in the far infra-red, are urgently needed for comparison with the radio data.

\section{References}

[1] Gaposchkin, S. I. Variable Stars, 10, 337, 1955.

[2] Oosterhoff, P. Th. Bull. Astr. Inst. Netherl. 13, 67, 1956.

[3] Stebbins, J. and Whitford, A. E. Astroph. J. 106, 235, 1947.

[4] Kaliniak, A. A., Krassovsky, V. I. and Nikonov, V. B. Dok. Akad. Nauk S.S.S.R. 66, 25, 1949.

[5] Dufay, J., Bigay, J. H. and Berthier, P. Vistas in Astronomy, 1539, 1956 (Pergamon Press, London).

[6] Haddock, F. I., Mayer, C. H. and Sloanaker, R. M. Astroph. J. 1x9, 456, 954.

[7] Mills, B. Y. Observatory, 76, 65, 1956.

[8] Davies, R. D. and Williams, D. R. W. Nature, 175, 1079, 1955.

[9] McClain, E. F. Astroph, J. 122, 376, 1955.

[10] Woerden, H. van, Rougoor, W. and Oort, J. H. C.R. 244, I691, 957.

[i I] Parenago, P. P. Uspekhi Astr. Nauk, 4, 69, $194^{8 .}$

\section{(E) THE GALAGTIG DISK}

According to the general evolutionary ideas outlined in section (A), p. 13 above, we should find in the disk both old objects, formed immediately after the contraction of the primeval cloud into the disk, and recently formed stars. The study of the interarm population of our Galaxy is a diffcult problem due to the interstellar absorption. Information from nearby galaxies, especially from $\mathrm{M}_{3} \mathrm{I}$, is in some ways more readily obtainable. 